\title{
Article \\ New Training Tasks for Stepwise Loading in Isometric Bodyweight Squat with Active Posture Control
}

\author{
Tetsuro Kitamura ${ }^{1,2,+}$, Yukako Ishida ${ }^{1,+}{ }^{\text {, Shinji Tsukamoto }}{ }^{1,2}{ }^{(\mathbb{D}}$, Manabu Akahane ${ }^{3}$, Tomoo Mano ${ }^{1}$, \\ Yasuyo Kobayashi ${ }^{1}$, Yasuhito Tanaka ${ }^{2}$ and Akira Kido ${ }^{1, *}$ \\ 1 Department of Rehabilitation Medicine, Nara Medical University, 840 Shijo-Cho, \\ Kashihara 634-8522, Nara, Japan; kitamuratetsurou@naramed-u.ac.jp (T.K.); \\ yukachan@naramed-u.ac.jp (Y.I.); shinji104@mail.goo.ne.jp (S.T.); manoneuro@naramed-u.ac.jp (T.M.); \\ tyasuyo@naramed-u.ac.jp (Y.K.) \\ 2 Department of Orthopedic Surgery, Nara Medical University, 840 Shijo-Cho, Kashihara 634-8521, Nara, Japan; \\ yatanaka@naramed-u.ac.jp \\ 3 Department of Health and Welfare Services, National Institute of Public Health, 2-3-6 Minami, \\ Wako-Shi 351-0197, Saitama, Japan; akahane.m.aa@niph.go.jp \\ * Correspondence: akirakid@naramed-u.ac.jp; Tel.: +81-744-223-051 \\ + The authors contributed equally.
}

Citation: Kitamura, T.; Ishida, Y.; Tsukamoto, S.; Akahane, M.; Mano, T.; Kobayashi, Y.; Tanaka, Y.; Kido, A. New Training Tasks for Stepwise Loading in Isometric Bodyweight Squat with Active Posture Control. Appl. Sci. 2021, 11, 8151. https:// doi.org/10.3390/app11178151

Academic Editor: Shin-Da Lee

Received: 8 July 2021

Accepted: 31 August 2021

Published: 2 September 2021

Publisher's Note: MDPI stays neutral with regard to jurisdictional claims in published maps and institutional affiliations.

Copyright: (c) 2021 by the authors. Licensee MDPI, Basel, Switzerland. This article is an open access article distributed under the terms and conditions of the Creative Commons Attribution (CC BY) license (https:/ / creativecommons.org/licenses/by/ $4.0 /)$.

\begin{abstract}
Isometric bodyweight squats are fundamental exercises in athletic training and rehabilitation. Previously, we measured muscle activity in a normal squat posture (NSP) and a squat posture with the center of foot pressure (COP) intentionally shifted forward as far as possible (forwardshifted posture: FSP), and the muscle activity patterns varied significantly according to the COP location. This study focused on stepwise loading as a training strategy. Ten healthy male participants performed isometric bodyweight squats in a previous study, adopting the NSP and FSP, with three knee flexion angles $\left(30^{\circ}, 60^{\circ}\right.$, and $\left.90^{\circ}\right)$. The muscle activities of the vastus medialis (VM), semitendinosus, tibialis anterior (TA), and gastrocnemius muscle lateral head were measured using surface electromyogram. This study further explored the relationship between COP shifting and knee flexion angles on electromyogram changes using three-dimensional diagrams. In one-way repeated measures analysis of variance by ranks, knee flexion angles affected the muscle activities of the VM and TA in the NSP and muscle activities of the VM in the FSP. Combining these findings, stepwise loading tasks were created to train individual target muscles. The ten male participants examined all the tasks, and the feasibility was confirmed accordingly.
\end{abstract}

Keywords: isometric bodyweight squat; center of pressure; electromyogram; rehabilitation; elderly

\section{Introduction}

The benefits of squat exercises have been reported in athletic training [1,2], rehabilitation [3,4], and locomotive syndrome in elderly people [5]. Squats can refer to a wide variety of exercises, comprising partial squat [6], half squat [7], parallel squat [8,9], full squat [8,9], and deep squat [7]. Furthermore, there are front [9-11], back [10-12], and overhead squats [12] with various bar positionings.

Among these, this study focused on the isometric bodyweight squat as a rehabilitation approach for elderly people, especially those with a risk of frailty. Isometric exercises provide moderate muscle strengthening without joint motion $[13,14]$, indicating gentle joint loading. A bodyweight squat can be performed with no equipment in a limited space. Considering that the squat exercise program is for elderly people, appropriate training intensity should be applied.

Previously, we measured muscle activity patterns in a normal isometric squat posture (NSP) and a squat posture with the center of foot pressure (COP) intentionally shifted forward as far as possible (forward-shifted posture: FSP), and varied muscle activity 
patterns were reported according to the COP location [15]. In this study, we focused on the muscle activities of the vastus medialis (VM), semitendinosus (ST), lateral head of the gastrocnemius (GL), and tibialis anterior (TA) muscles. Because these are the main components of lower limb kinetics and kinematics, their activities reflect the training loads for each muscle. Our electromyogram (EMG) data revealed that precise COP control by the participants could successfully change the squat exercise load. Next, it was assumed that a sequential combination of several postures could be a training task with stepwise loading for specific target muscles. Previously, a deeper squat was inconclusively considered to increase the squat exercise load, but this has recently been invalidated [15]. Muscle activity showed a significant change with COP shifting at specific knee flexion angles; however, the angles were different in target muscles (e.g., $30^{\circ}, 60^{\circ}$, and $90^{\circ}$ for TA and $30^{\circ}$ and $60^{\circ}$ for ST). Finally, it was concluded that active control of the COP location during squats could be a new training approach targeting specific muscle groups [15]. This pilot study further explored the relationship between COP shifting and knee flexion angles on EMG changes using three-dimensional (3D) diagrams based on the datasets. This study aimed to create new training tasks with stepwise loading for each target muscle.

\section{Materials and Methods}

\subsection{Subjects}

Ten healthy male patients with no history of knee injury, knee pain, or surgery participated in this study. All participants had previous experience of performing squats. The participants' average age was 26.9 years (standard deviation $(\mathrm{SD})=2.2$ ), average height was $1.70 \mathrm{~m}(\mathrm{SD}=0.5)$, and average body mass was $70.2 \mathrm{~kg}(\mathrm{SD}=11.1)$. The content of the experiment was sufficiently explained to the subjects, and only those who voluntarily agreed to participate in the experiment were enrolled. All subjects provided informed consent for the study. Participation was voluntary and agreed on. The study was conducted in accordance with the principles of the Declaration of Helsinki and the laws and regulations of Japan. This study was approved by the Ethics Committee of Nara Medical University (No. 1969). No subjects had difficulty holding the squat position for $5 \mathrm{~s}$. Each task was performed in triplicate.

\subsection{EMG Datasets of Squat Postures and Experimental Approach}

Previously, we investigated the muscle activity pattern in the NSP and FSP at three flexion angles of the knee joint $\left(30^{\circ}, 60^{\circ}\right.$, and $\left.90^{\circ}\right)$ (Figure 1) [15]. The COP location was measured using a stabilometer (Twin Gravicorder G-6100; Anima Corp., Tokyo, Japan). The participants visually checked the COP during squats. According to Perotto's method [16], the muscle activities of the right VM, ST, GL, and TA were measured using the MWATCH101 (Wada Aircraft Technology CO. Ltd. Aichi, Japan). After the statistical evaluation, stepwise loading tasks were performed for each muscle training.

\subsection{Designing New Training Tasks for Stepwise Loading}

Based on the previous data on COP shifting, new training tasks were designed. The tasks were sequential combinations of isometric squat postures that were designed to significantly increase the load (Figure 2). The participants held each posture for one minute and switched to the next posture. The participants controlled their posture by selfmonitoring a stabilometer. Knee flexion was assisted by an assistant using a goniometer with an acceptable range of $\pm 5^{\circ}$. Muscle activity of the lower limbs was measured and further analyzed. 

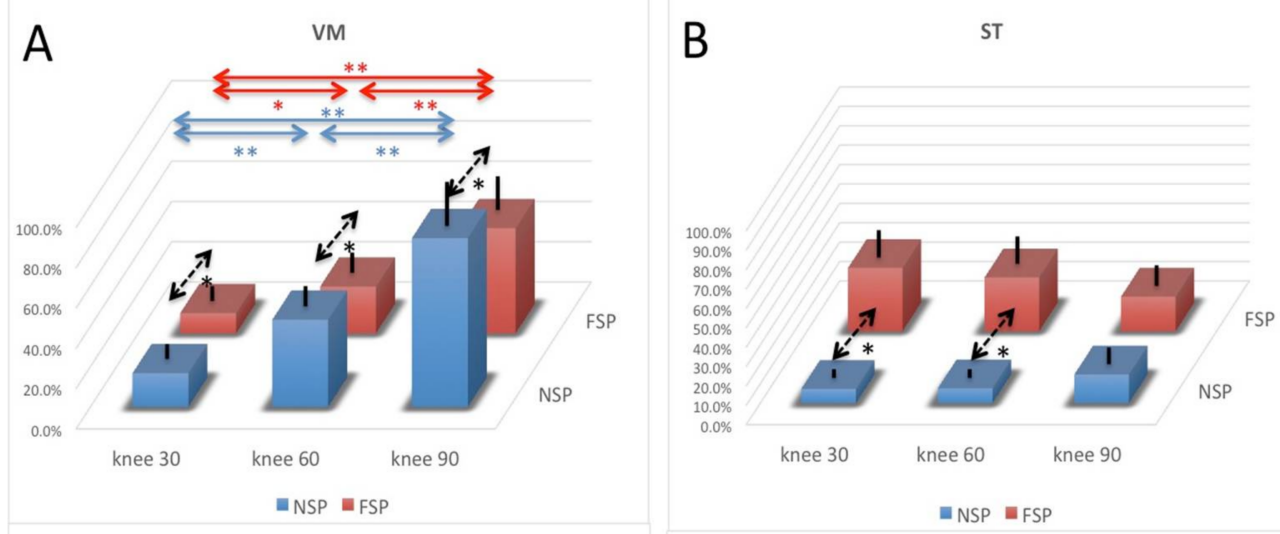

C

TA

D

GL
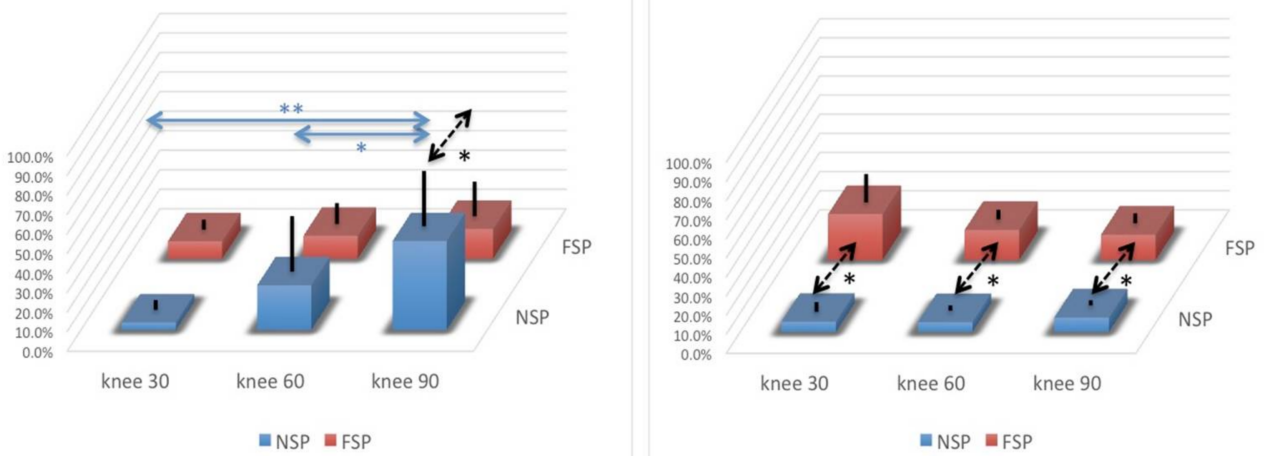

Figure 1. Muscle activity compared at different knee flexion angles in the FSP and NSP. Muscle activity is compared at different knee flexion angles of $30^{\circ}, 60^{\circ}$, and $90^{\circ}$ in the FSP and NSP. The measured muscles are vastus medialis (VM, (A)), semitendinosus (ST, (B)), tibialis anterior (TA, (C)), and gastrocnemius muscle lateral head (GL, (D)). Muscle activity is normalized using the integrated EMG at maximum voluntary isometric contraction. Black arrows show the differences between the FSP and NSP, which were previously reported. * Significant difference $(p<0.05)$ for multiple comparisons. ** Significant difference $(p<0.01)$.

A

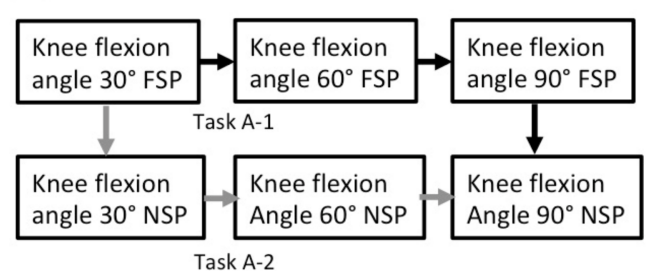

C

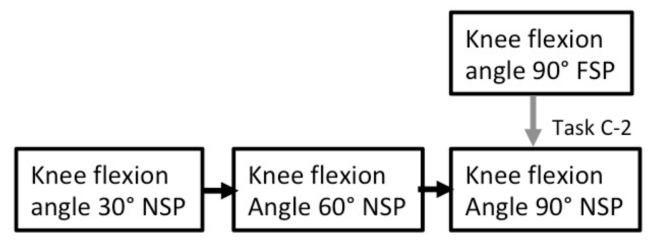

B

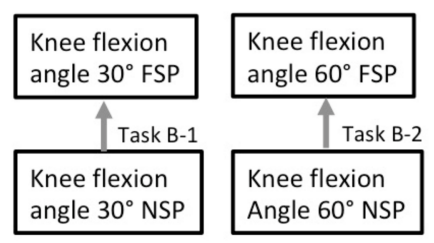

D

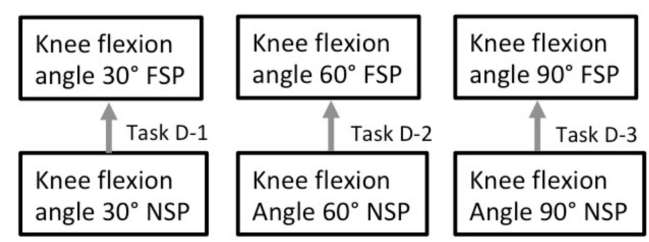

Figure 2. New training tasks. The training tasks are sequential combinations of the isometric squat postures designed to significantly increase the load. Participants hold each posture for $1 \mathrm{~min}$ and the muscle activities and the COP are measured accordingly. The targeted muscles are VM (A), ST (B), TA(C), and GL (D). 


\subsection{Statistical Analysis}

Statistical analyses were performed using Statcel 4 (OMS Publishing Inc., Saitama, Japan) [17] and GPower software 3.1 (University of Dusseldorf, Dusseldorf, Germany). Statistical significance was set at $p<0.05$. Sample size determination was performed using the GPower software prior to data collection (power $=0.8$ ). After data collection, the power for all variables with group parameters (mean, SD, and correlation) was confirmed. Post hoc analysis was performed using the Wilcoxon signed-rank test. The determined effect sizes were calculated using the group parameters. The Friedman test was used to evaluate differences in the EMG data of each evaluated knee joint angle $\left(30^{\circ}, 60^{\circ}\right.$, and $\left.90^{\circ}\right)$ because the EMG data were dependent and nonparametric. Friedman's test's null hypothesis was rejected; thus, multiple comparison analyses were performed using Scheffe's method.

\section{Results}

3.1. Lower Limb Muscle Activities at Knee Flexion Angles of $30^{\circ}, 60^{\circ}$, and $90^{\circ}$ in the FSP and NSP

Figure 1 shows the muscle activity at the three knee flexion angles in the FSP and NSP. In one-way repeated measures analysis of variance by ranks, the knee flexion angle induced significant changes in VM muscle activity in the NSP and FSP (Figure 1A) and TA muscle activity in the NSP (Figure 1C). No significant differences were found in the ST muscle activity (Figure 1B) and GL muscle activity (Figure 1C). The black arrows illustrate the differences between the NSP and FSP (Figure 1A-D), as reported in a previous study [15]. In the multiple comparison analyses, significant differences were found in VM muscle activity at $30^{\circ}$ vs. $60^{\circ}\left(p<0.05\right.$, in the FSP and $p<0.01$, in the NSP), at $30^{\circ}$ vs. $90^{\circ}(p<0.01$ in the FSP and the NSP), and $60^{\circ}$ vs. $90^{\circ}$ in the FSP and the NSP $(p<0.01$ in the FSP and the NSP). Furthermore, significant differences were found in TA muscle activity at $30^{\circ}$ vs. $90^{\circ}$ in the NSP $(p<0.01)$ and $60^{\circ}$ vs. $90^{\circ}$ in the NSP $(p<0.05)$ (Figure 1C).

\subsection{Feasibility of New Training Tasks for Stepwise Loading}

Figure 2 shows the training tasks, which are sequential combinations of isometric squat postures designed to significantly increase the load. Briefly, a route was created from the red, blue, and black arrows in Figure 1 to obtain stepwise loading. Figure 3 shows a representative scheme for training task A-1. Ten patients participated in this study. None of the participants reported difficulty in maintaining posture or following tasks. Significant EMG changes were found in the targeted muscles in a stepwise manner, which is consistent with previous datasets (data not shown).

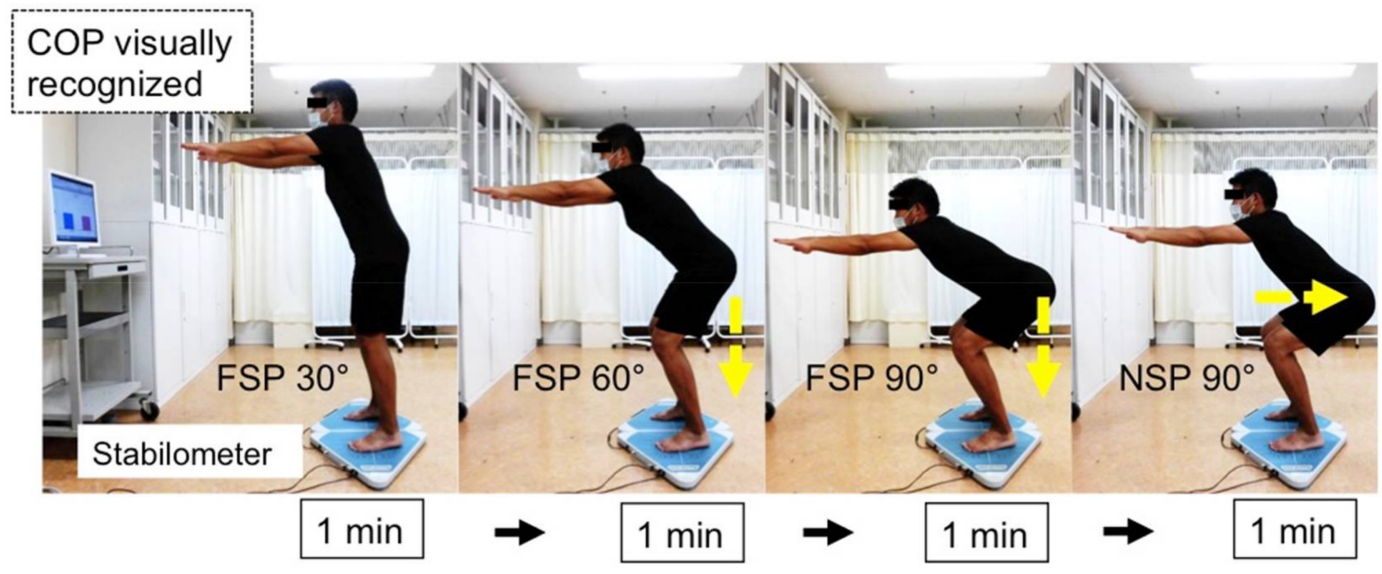

Figure 3. Representative scheme of training task A-1. Participants control the posture by monitoring by a stabilometer. Knee flexion is assisted by an assistant using a goniometer. The participants hold the posture for $1 \mathrm{~min}$ and switch to the next posture afterwards. Muscle activities of lower limbs are recorded and evaluated. 


\section{Discussion}

The influence of changing the knee flexion angles on muscle activity in bodyweight squats has been previously reported in several studies [18-21]. However, in most studies, the subjects used equipment such as weight bars [19] or decline boards [18], or were analyzed on unstable floors [20,21]. The COP (or center of gravity) was not controlled in any of the studies. Contemporarily, muscle activity in isometric bodyweight squats has rarely been analyzed. Previously, muscle activity patterns were reported to vary significantly according to the COP location in the squat posture [15]. Precise control of the COP is essential for designing training tasks for bodyweight squats. In this study, the impact of changing the knee flexion angles on muscle activity patterns was analyzed using previous datasets; thus, new training tasks were designed for stepwise loading. First, heterogeneous effects of knee flexion on muscle activity patterns were found in bodyweight squats under precise COP control. For example, VM muscle activity increased the load from the FSP to the NSP at knee flexion angles of $90^{\circ}$, while the GL muscle activity decreased under the same conditions. Furthermore, deeper squats do not always provide a higher load to the lower limb muscles (Figure 1B-D).

Isometric exercises provide moderate muscle strengthening without joint motion $[13,14]$, and a bodyweight squat can be performed in a limited space without any equipment. Additionally, it can be performed at home without supervision. Therefore, it is suggested that isometric bodyweight squats could be an ideal training approach for elderly people. In a recent study, the effect of mechanically assisted squat exercise on lung function in older females with sarcopenia was reported [22]. Furthermore, an assisted squat may be another possible approach for elderly people. However, the muscle activity characteristics in a simple squat posture should be investigated comprehensively before starting the assisted approach.

In the experiments, the participants could confirm the COP shifting by themselves, and assistance was needed to maintain the knee flexion angles. It would be ideal for active posture control if the participants could also visually ensure knee flexion angles using some devices. This pilot study has some limitations, including the sample size, sex, and training background of the participants. Further investigation of the appropriate training intensity should be considered as it is essential to modulate it by the number of training tasks for individual conditions. Stepwise loading may relieve the impact for elderly people, especially those at risk of frailty.

\section{Conclusions}

In this study, lower limb muscle activities varied according to the knee flexion angle in the FSP or NSP. New training tasks were designed, and the feasibility based on the differences in muscle activities in the datasets was examined. All participants completed the tasks successfully. As expected from the datasets, significant EMG changes were confirmed in the targeted muscles in a stepwise manner. The new training tasks of isometric bodyweight squats could be useful for elderly people in various clinical situations in which closed kinetic chain exercise should be used on careful physical examination for fatigue or pain in each muscle group.

Author Contributions: Conceptualization, T.K. and Y.I.; methodology, T.K.; software, S.T.; validation, S.T. and M.A.; formal analysis, Y.I., Y.K. and T.M.; resources, T.K.; data curation, Y.I. and M.A.; writing—original draft preparation, T.K. and Y.I.; writing—review and editing, A.K.; visualization, Y.I.; supervision, A.K.; project administration, A.K. and Y.T.; funding acquisition, Y.I. and A.K. All authors have read and agreed to the published version of the manuscript.

Funding: This work was supported by JSPS KAKENHI (grant numbers JP24592241, JP18K10753, JP15K01381, and JP20K19383) and by the World Health Organization Centre for Health Development (grant number WHO Kobe Centre-WKC: K18007).

Institutional Review Board Statement: The study was conducted according to the guidelines of the Declaration of Helsinki, and approved by the ethics committee of Nara Medical University (No.1969). 
Informed Consent Statement: Informed consent was obtained from all subjects involved in the study.

Data Availability Statement: The data that support the findings of this study are available from the corresponding author, A.K., upon reasonable request.

Conflicts of Interest: The authors declare no conflict of interest.

\section{References}

1. Grooms, D.R.; Palmer, T.; Onate, J.A.; Myer, G.D.; Grindstaff, T. Soccer-specific warm-up and lower extremity injury rates in collegiate male soccer players. J. Athl. Train. 2013, 48, 782-789. [CrossRef] [PubMed]

2. Flocco, P.; Galeoto, G. Effect of blood flow restriction training on physiological outcomes in healthy athletes: A systematic review and meta-analysis. MLTJ 2021, 11, 101-117. [CrossRef]

3. Escamilla, R.F.; Fleisig, G.S.; Lowry, T.M.; Barrentine, S.W.; Andrews, J.R. A three-dimensional biomechanical analysis of the squat during varying stance widths. Med. Sci. Sports Exerc. 2001, 33, 984-998. [CrossRef] [PubMed]

4. Miccio, S.; Berardi, A.; Tofani, M.; Galeoto, G. Conservative rehabilitation treatments of iliotibial band syndrome: A systematic review. MLTJ 2021, 11, 29-40. [CrossRef]

5. Nakamura, K. The concept and treatment of locomotive syndrome: Its acceptance and spread in Japan. J. Orthop. Sci. 2011, 16, 489-491. [CrossRef] [PubMed]

6. $\quad$ Escamilla, R.F.; Fleisig, G.S.; Zheng, N.; Lander, J.E.; Barrentine, S.W.; Andrews, J.R.; Moorman, C.T., 3rd. Effects of technique variations on knee biomechanics during the squat and leg press. Med. Sci. Sports Exerc. 2001, 33, 1552-1566. [CrossRef] [PubMed]

7. Hartmann, H.; Wirth, K.; Klusemann, M. Analysis of the load on the knee joint and vertebral column with changes in squatting depth and weight load. Sports Med. 2013, 43, 993-1008. [CrossRef] [PubMed]

8. Caterisano, A.; Moss, R.F.; Pellinger, T.K.; Woodruff, K.; Lewis, V.C.; Booth, W.; Khadra, T. The effect of back squat depth on the EMG activity of 4 superficial hip and thigh muscles. J. Strength Cond. Res. 2002, 16, 428-432. [PubMed]

9. Contreras, B.; Vigotsky, A.D.; Schoenfeld, B.J.; Beardsley, C.; Cronin, J. A comparison of gluteus maximus, biceps femoris, and vastus lateralis electromyography amplitude in the parallel, full, and front squat variations in resistance-trained females. J. Appl. Biomech. 2016, 32, 16-22. [CrossRef] [PubMed]

10. Aspe, R.R.; Swinton, P.A. Electromyographic and kinetic comparison of the back squat and overhead squat. J. Strength Cond. Res. 2014, 28, 2827-2836. [CrossRef]

11. Clark, D.R.; Lambert, M.I.; Hunter, A.M. Muscle activation in the loaded free barbell squat: A brief review. J. Strength Cond. Res. 2012, 26, 1169-1178. [CrossRef] [PubMed]

12. Gullett, J.C.; Tillman, M.D.; Gutierrez, G.M.; Chow, J.W. A biomechanical comparison of back and front squats in healthy trained individuals. J. Strength Cond. Res. 2009, 23, 284-292. [CrossRef]

13. Iversen, M.D.; Kale, M.K. Physical therapy management of select rheumatic conditions in older adults. In Geriatric Rheumatology: A Comprehensive Approach; Nakasato, Y., Yung, R., Eds.; Springer Science and Business Media: Heidelberg, Germany, 2011; pp. 101-102.

14. Kaufmann, T.L.; Bolton, M. Muscle weakness and therapeutic exercise. In Comprehensive Guide to Geriatric Rehabilitation; Kauffman, T.L., Scott, R., Barr, J.O., Moran, M.L., Eds.; Elsevier Health Sciences: New York, NY, USA, 2014; pp. 107-114.

15. Kitamura, T.; Kido, A.; Ishida, Y.; Kobayashi, Y.; Tsukamoto, S.; Tanaka, T. Muscle activity pattern with a shifted center of pressure during the squat exercise. J. Sports Sci. Med. 2019, 17, 248-252.

16. Perotto, A.O. Anatomical Guide for the Electromyographer: The Limbs and Trunk; Charles C. Thomas Publisher: Springfield, IL, USA, 1994.

17. Yanai, H. Statcel: The Useful Add-in Software Forms on Excel, 4th ed.; OMS Publishing, Inc.: Tokyo, Japan, 2015.

18. Lee, D.; Lee, S.; Park, J. Impact of decline-board squat exercises and knee joint angles on the muscle activity of the lower limbs. J. Phys. Ther. Sci. 2015, 27, 2617-2619. [CrossRef] [PubMed]

19. Marchetti, P.H.; Jarbas da Silva, J.; Jon Schoenfeld, B.; Nardi, P.S.; Pecoraro, S.L.; D'Andréa Greve, J.M.; Hartigan, E. Muscle activation differs between three different knee joint-angle positions during a maximal isometric back squat exercise. J. Sports Med. 2016, 2016, 3846123. [CrossRef] [PubMed]

20. Han, D.; Nam, S.; Song, J.; Lee, W.; Kang, T. The effect of knee flexion angles and ground conditions on the muscle activation of the lower extremity in the squat position. J. Phys. Ther. Sci. 2017, 29, 1852-1855. [CrossRef]

21. Kang, J.I.; Park, J.S.; Choi, H.; Jeong, D.K.; Kwon, H.M.; Moon, Y.J. A study on muscle activity and ratio of the knee extensor depending on the types of squat exercise. J. Phys. Ther. Sci. 2017, 29, 43-47. [CrossRef]

22. Jeon, Y.K.; Shin, M.J.; Kim, C.M.; Lee, B.J.; Kim, S.H.; Chae, D.S. Effect of squat exercises on lung function in elderly women with sarcopenia. J. Clin. Med. 2018, 7, 167. [CrossRef] [PubMed] 